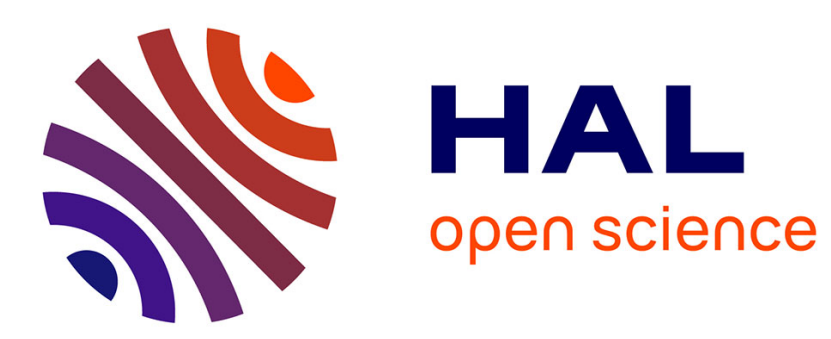

\title{
Parthénocarpie naturelle chez la tomate. II. - Etude d'une collection variétale
}

\author{
Jacqueline Philouze, Maguy Milesi
}

\section{To cite this version:}

Jacqueline Philouze, Maguy Milesi. Parthénocarpie naturelle chez la tomate. II. - Etude d'une collection variétale. Agronomie, 1985, 5 (1), pp.47-54. hal-00884732

\section{HAL Id: hal-00884732 \\ https://hal.science/hal-00884732}

Submitted on 1 Jan 1985

HAL is a multi-disciplinary open access archive for the deposit and dissemination of scientific research documents, whether they are published or not. The documents may come from teaching and research institutions in France or abroad, or from public or private research centers.
L'archive ouverte pluridisciplinaire HAL, est destinée au dépôt et à la diffusion de documents scientifiques de niveau recherche, publiés ou non, émanant des établissements d'enseignement et de recherche français ou étrangers, des laboratoires publics ou privés. 


\title{
Parthénocarpie naturelle chez la tomate. II. - Etude d'une collection variétale
}

\author{
Jacqueline PHILOUZE \\ avec la collaboration technique de Maguy MiLesı \\ I.N.R.A., Station d'Amélioration des Plantes marấchères \\ Centre de Recherches d'A vignon, B.P. 94, F 84140 Montfavet
}

RÉSUMÉ

L'étude a porté sur 10 variétés pour juger de leur aptitude à la parthénocarpie naturelle, ainsi que sur une variété témoin non parthénocarpique, "Monalbo ». Quatre essais ont été réalisés en culture de printemps sous serre verre chauffée, avec floraison en mars-avril. Deux traitements ont été appliqués : d'une part castration des fleurs, non suivie de pollinisation ; d'autre part pollinisation, soit manuelle après castration, soit naturelle sans castration. On a effectué l'étude comparée des pourcentages de nouaison et des poids des fruits à la suite des 2 types de traitements, l'étude de la corrélation entre le poids des fruits et leur nombre de graines à la suite des traitements avec pollinisation, ainsi que des observations sur la précocité et la rapidité du grossissement parthénocarpique de l'ovaire à la suite du traitement sans pollinisation, et enfin des observations sur l'aspect extérieur des fruits.

Ces critères ont permis de classer les variétés selon leur aptitude à la parthénocarpie :

- Aptitude marquée à la parthénocarpie : "Severianin », " Montfavet 191 » et «75/59 ».

- Aptitude relativement limitée à la parthénocarpie : « Pridneprovskij », « Earlinorth », « Parteno », « Oregon T 5-4 ", " Lyconorma " et " Lycoprea ".

- Aptitude nulle ou quasi nulle à la parthénocarpie : "Sub-Arctic Plenty » et " Monalbo ».

Mot clé additionnel : Lycopersicon esculentum.

\begin{abstract}
Natural parthenocarpy in tomato. II. - Study of a varietal collection
Ten varieties were studied in order to estimate their capacity for natural parthenocarpy, in comparison with a control variety, "Monalbo », which is not parthenocarpic. Four experiments were carried out in spring cultivation under a heated glasshouse, the flowering taking place in March and April. Two treatments were used : on the one hand, emasculation of the flowers, not followed by pollination; on the other hand, pollination, either hand pollination after emasculation, or open pollination without emasculation. Comparisons were made between the percentages of fruit set and the average fruit weights for the 2 treatments; the correlation between the fruit weight and the number of seeds was calculated for the treatments with pollination; observations were made on the earliness and the speed of parthenocarpic development of the ovary for the treatment without pollination, and on the external appearance of the fruits. These criteria were used to classify the varieties according to their capacity for parthenocarpy :

- marked capacity for parthenocarpy : "Severianin", "Montfavet 191" and "75/59" ;

— relatively limited capacity for parthenocarpy : “Pridneprovskij", "Earlinorth", "Parteno", “Oregon T 5-4", "Lyconorma" and "Lycoprea" ;

- little or no capacity for parthenocarpy : "Sub-Arctic Plenty" and "Monalbo".
\end{abstract}

Additional key word : Lycopersicon esculentum

\section{INTRODUCTION}

Certaines variétés de tomate manifestent une aptitude à la parthénocarpie naturelle susceptible d'être utilisée dans la pratique. C'est ce qu'ont montré plusieurs auteurs, en testant du matériel végétal diversifié, dans des conditions expérimentales très variées, avec des méthodes différentes pour apprécier l'aptitude à la parthénocarpie. Une revue bibliographique sur le sujet a été faite par PHILOUZE (1983). L'observation, dans des conditions de milieu et avec des méthodes comparables, d'une collection de variétés signalées dans la littérature comme ayant une aptitude marquée à la parthénocarpie, s'avère être un préalable indispensable avant toute étude plus approfondie du phènomène de parthénocarpie naturelle chez la tomate. Ce sont les résultats de l'observation d'une telle collection qui sont présentés ici. 


\section{MATÉRIEL ET MÉTHODES}

\section{A. Matériel végétal}

Les variétés dont la liste est donnée dans le tableau 1 ont été observées.

\section{Matériel en provenance d'Allemagne fédérale}

Ce matériel a été très étudié au Bundesforschungsanstalt für Gartenbauliche Pflanzenzüchtung, à Ahrensburg, en particulier par REIMANN-PHILIPP \& FUCHS (1969), PREIL \& REIMANN-PHILIPP (1969) et PREIL (1973).

- «75/59», lignée F 13 issue du croisement «Atom » $\times$ « Bubjekosoko » (REIMANN-PHILIPP, comm. pers., 1977).

- «Lyconorma » et « Lycoprea », variétés issues du croisement «Priora » $\times$ «Heinemanns Jubiläum » (REIMANN-PHILIPP, comm. pers., 1977).

\section{Matériel en provenance d'Union Soviétique}

- "Pridneprovskij ». Cette variété, sélectionnée en Ukraine, a été étudiée en Moldavie par LUDNIKOVA (1970). Nous avons travaillé avec un lot de «Pridneprovskij » reçu des Pays-Bas et portant la référence IVT 771249.

- «Severianin ». Cette variété a été étudiée à l'Académie Timiriazev de Moscou, en particulier par DOVEDAR (1973).

\section{Matériel d'origines diverses}

- «Oregon T 5-4 », sélectionnée aux Etats-Unis. BAGGETT \& FRAZIER (1978) ont montré que cette variété avait tendance à former des fruits parthénocarpiques dans les conditions de température fraîche de l'été à Corvallis $\left(9^{\circ} 4\right.$ à $11^{\circ} \mathrm{C}$ la nuit en juin-juillet) ; par contre il n'y avait pas formation de fruits parthé- nocarpiques en serre où la température nocturne était de l'ordre de $18^{\circ} \mathrm{C}$. La variété «Oregon T 5-4» manque d'uniformité, car il s'agit d'un mélange de 10 lignées $\mathrm{F} 10$ provenant de 2 familles $\mathrm{F} 9$ voisines, mais différentes (BAGGETT \& FRAZIER, 1978). Le matériel que nous avons reçu était donc hétérogène : pour la forme des fruits, d'ovales lisses réguliers à plats côtelés irréguliers et pour le nombre de loges; les plantes que nous avons retenues pour cette étude portaient des fruits ovales à 2-3 loges.

- «Sub-Arctic Plenty » (= BL 6807), sélectionnée au Canada pour son aptitude à nouer à basses températures (HARRIS, 1972). EL AHMADI \& STEVENS (1979) ont montré que la majorité des fruits formés à températures élevées ne contenaient pas de graines.

- «Earlinorth », sélectionnée au Canada, est capable de former en fécondation libre des fruits avec peu ou pas de graines.

- «Parteno », étudiée en Pologne par PASZKOWSKA (1982). Cette variété a été sélectionnée à partir du croisement "Earlinorth 》 $\times$ «Coldset " (PASZKOWSKA, comm. pers., 1979).

- « Montfavet 191 », mutant apparu naturellement dans une de nos lignées en sélection. Ce mutant a des anthères courtes et montre une aptitude à la parthénocarpie naturelle, caractéristiques que SORESSI \& SALAMINI (1975) attribuent à 2 gènes différents : sha (short anthers) et pat (parthenocarpic), et que PECAUT \& PHILOUZE (1978) attribuent à un seul gène.

- Enfin nous avons introduit en guise de témoin la variété non parthénocarpique «Monalbo".

Nous donnons dans le tableau 1 quelques caractéristiques concernant ces variétés : type de croissance (indéterminée ou déterminée), présence ou absence d'un collet vert sur le fruit avant maturité, nombre de loges des fruits. Notons enfin que « Lycoprea » a des feuilles de pomme de terre (gène $c$ ), « Pridneprovskij » des feuilles entières (gène $e$ ) et «Oregon T 5-4 » des fruits roses (gène $y$ ).

TABLEAU

Variétés observées.

Varieties observed.

\begin{tabular}{|c|c|c|c|c|c|c|c|c|}
\hline \multirow[b]{2}{*}{ Variétés } & \multirow[b]{2}{*}{ Origine } & \multicolumn{3}{|c|}{ Caractéristiques variétales } & \multicolumn{4}{|c|}{ Essais réalisés } \\
\hline & & $\begin{array}{c}\text { type } \\
\text { croissance } \\
\left({ }^{1}\right)\end{array}$ & $\begin{array}{l}\text { collet avant } \\
\text { maturité } \\
\left(^{2}\right)\end{array}$ & $\begin{array}{c}\text { nombre } \\
\text { loges } \\
\left(^{3}\right)\end{array}$ & $\begin{array}{c}\text { Essai } \\
1\end{array}$ & $\begin{array}{c}\text { Essai } \\
2\end{array}$ & $\begin{array}{c}\text { Essai } \\
3\end{array}$ & $\begin{array}{c}\text { Essai } \\
4\end{array}$ \\
\hline$《 75 / 59 »$ & Allemagne fédérale & $\mathrm{sp}$ & $\mathbf{u}$ & $2-4$ & + & + & + & \\
\hline “ Lyconorma " & Allemagne fédérale & $\mathrm{sp}$ & + & $3-6$ & & + & & \\
\hline « Lycoprea » & Allemagne fédérale & $\mathrm{sp}$ & + & $3-6$ & & + & & \\
\hline « Pridneprovskij » & Ukraine, U.R.S.S. & $\mathrm{sp}$ & + & nbses & + & & & + \\
\hline « Severianin » & Russie, U.R.S.S. & $\mathrm{sp}$ & + & nbses & + & + & + & \\
\hline «Oregon T 5-4» & Oregon, U.S.A. & $\mathrm{sp}$ & $\mathbf{u}$ & $2-3$ & + & & & \\
\hline "Sub-Arctic Plenty " & Alberta, Canada & $\mathrm{sp}$ & $\mathbf{u}$ & $2-4$ & & + & & \\
\hline « Earlinorth » & Alberta, Canada & $\mathrm{sp}$ & $\mathbf{u}$ & nbses & + & & & \\
\hline «Parteno » & Pologne & $\mathrm{sp}$ & $\mathrm{u}$ & nbses & & + & + & \\
\hline “ Montfavet 191 » & France (I.N.R.A.) & + & $\mathbf{u}$ & $2-3$ & & & + & \\
\hline «Monalbo » & France (I.N.R.A.) & + & $\mathbf{u}$ & $2-3$ & & & + & \\
\hline
\end{tabular}

(1) Type de croissance : $+=$ indéterminée ; $\mathrm{sp}=$ déterminée.

(2) Collet avant maturité : $+=$ présence d'un collet vert ; $u=$ fruit de couleur uniforme.

( $\left.{ }^{3}\right)$ Loges nombreuses (nbses) : de 3 ou 4 à plus de 10 


\section{B. Méthodes}

\section{Techniques culturales}

Quatre essais ont été conduits au printemps sous serre verre chauffée, en conditions non limitantes de lumière et de température, au cours de 4 années différentes (tabl. 2). Les essais 1 et 2 ont été conduits en pleine terre, les essais 3 et 4 sur des plantes cultivées en pots. La période de floraison sur les 4 bouquets étudiés s'est échelonnée en mars et en avril pour l'ensemble des 4 essais.

\section{Techniques expérimentales}

Plusieurs traitements ont été appliqués :

- les fleurs ont été castrées avant la maturité du pollen ; aucun apport de pollen n'a été effectué sur le stigmate de ces fleurs (essais 1-2-3-4) ;

- les fleurs ont été castrées et pollinisées avec du pollen de "Apédice » (lignée riche en pollen de bonne qualité) (essai 3) ou du pollen de la même variété que le parent femelle (le traitement correspond donc à une autofécondation) (essais 1 et 2). Dans un cas comme dans l'autre, la pollinisation est effectuée à 2 reprises, le jour de la castration et 2 ou $3 \mathrm{j}$ après, avec du pollen prélevé sur des plantes cultivées dans les mêmes conditions que les plantes femelles;

- les fleurs, non castrées, ont été laissées libres de s'autoféconder naturellement (essai 4).

Ces traitements ont été appliqués sur 2 à 4 plantes, et sur 4 bouquets par plante ; les bouquets ont été limités à 8 fleurs au maximum.

Pour chaque traitement, le pourcentage de nouaison a été calculé. Tous les fruits, même très petits, qui se sont développés et ont atteint la maturité ont été considérés comme noués. Le poids minimum des fruits noués est variable selon les variétés. Dans nos essais, il a été de $6 \mathrm{~g}$ pour " 75/59 », "Lyconorma » et « Oregon T 5-4 », de 11 g pour «Lycoprea ", de 18 à $20 \mathrm{~g}$ pour " Parteno ", « Montfavet 191 » et " Monalbo ", de $30 \mathrm{~g}$ et plus pour les autres variétés. Tous les fruits ont été récoltés et pesés individuellement. De plus, pour les traitements avec pollinisation, les graines contenues dans chaque fruit ont été comptées (essais 2, 3 et 4); dans l'essai 1, les fruits ont sim- plement été classés en fruits ne contenant aucune graine et fruits contenant des graines.

Il a été ainsi possible de comparer les pourcentages de fruits noués à la suite des traitements avec et sans pollinisation. Les comparaisons entre les poids des fruits obtenus à la suite de ces 2 types de traitements ont été effectuées au moyen du test $t$ de Student. Des observations ont porté par ailleurs sur la précocité et la vitesse de grossissement des ovaires, ainsi que sur l'aspect général des fruits (fruits lisses ou plus ou moins côtelés, fruits brillants ou ternes).

Pour les fruits obtenus à la suite des traitements avec pollinisation, les corrélations entre le poids des fruits et le nombre de graines qu'ils contiennent ont été calculées (essais 2, 3 et 4). Dans les essais 1 et 4 , le poids des fruits contenant des graines a été comparé, au moyen du test $t$ de Student, à celui des fruits sans graines quand ces derniers représentaient au moins 10 p. $100 \mathrm{du}$ total.

\section{RÉSULTATS}

Les résultats des essais $1,2,3$ et 4 sont donnés dans les tableaux 3, 4, 5 et 6 respectivement.

Des fruits se sont développés sur toutes les variétés, pour chacun des traitements avec ou sans pollinisation dans les 4 essais, mais le nombre, la taille, l'aspect, la vitesse de grossissement des fruits issus du traitement sans pollinisation, en comparaison avec les fruits issus des traitements avec pollinisation, sont très différents selon les variétés. Nous présentons les variétés par ordre décroissant d'aptitude à la parthénocarpie naturelle, en nous basant pour ce classement sur le poids des fruits du traitement sans pollinisation, exprimé en pourcentage du poids des fruits des traitements avec pollinisation.

- «Severianin" (essais 1, 2, 3; tabl. 3, 4, 5) est la variété qui montre l'aptitude la plus élevée à la parthénocarpie, mais cette aptitude est variable selon les essais. Dans tous les cas, le déclenchement du grossissement des ovaires après le traitement sans pollinisation est précoce et l'aspect général des fruits (forme, couleur) est le même que celui des fruits issus du traitement avec pollinisation. Dans les essais 1 et 3 , le poids des fruits obtenus sans pollinisation n'est pas

TABLEAU 2

Techniques culturales et expérimentales pour 4 essais réalisés au printemps sous serre verre chauffée. Cultural and experimental techniques for 4 experiments carried out in the spring under a heated glasshouse.

\begin{tabular}{|c|c|c|c|c|}
\hline & Essai 1 & Essai 2 & Essai 3 & Essai 4 \\
\hline \multicolumn{5}{|l|}{ Techniques culturales } \\
\hline date semis & 29 déc. & 28 déc. & 22 jan. & 28 fév. \\
\hline date repiquage & 18 jan. & 22 jan. & 9 fév. & 17 mars \\
\hline date plantation ou rempotage & 16 fév. & 18 fév. & 6 mars & 11 avr. \\
\hline type de culture & pleine terre & pleine terre & pots & pots \\
\hline période de castration ( 4 bouquets) & 5 mars $\rightarrow 26$ mars & 3 mars $\rightarrow 28$ mars & 27 mars $\rightarrow 17$ avril & 12 avril $\rightarrow 2$ mai \\
\hline \multicolumn{5}{|l|}{ Nombre de plantes par traitement } \\
\hline fleurs castrées, pas de pollinisation & 2 & 3 & 4 & 3 \\
\hline \multicolumn{5}{|l|}{ fleurs castrées, pollinisation manuelle } \\
\hline - pollinisation par « Apédice » & - & - & 2 & - \\
\hline - autopollinisation & 2 & 2 ou 3 & - & - \\
\hline fleurs non castrées, autopollinisation naturelle & - & - & - & 2 \\
\hline
\end{tabular}


TABLEAU 3

Essai I. Culture de printemps, sous serre verre chauffée, en pleine terre. Période de castration : 5 au 26 mars.

Experiment 1. Spring cultivation, under a heated glasshouse, in soil. Period of emasculation: 5th to 26th March.

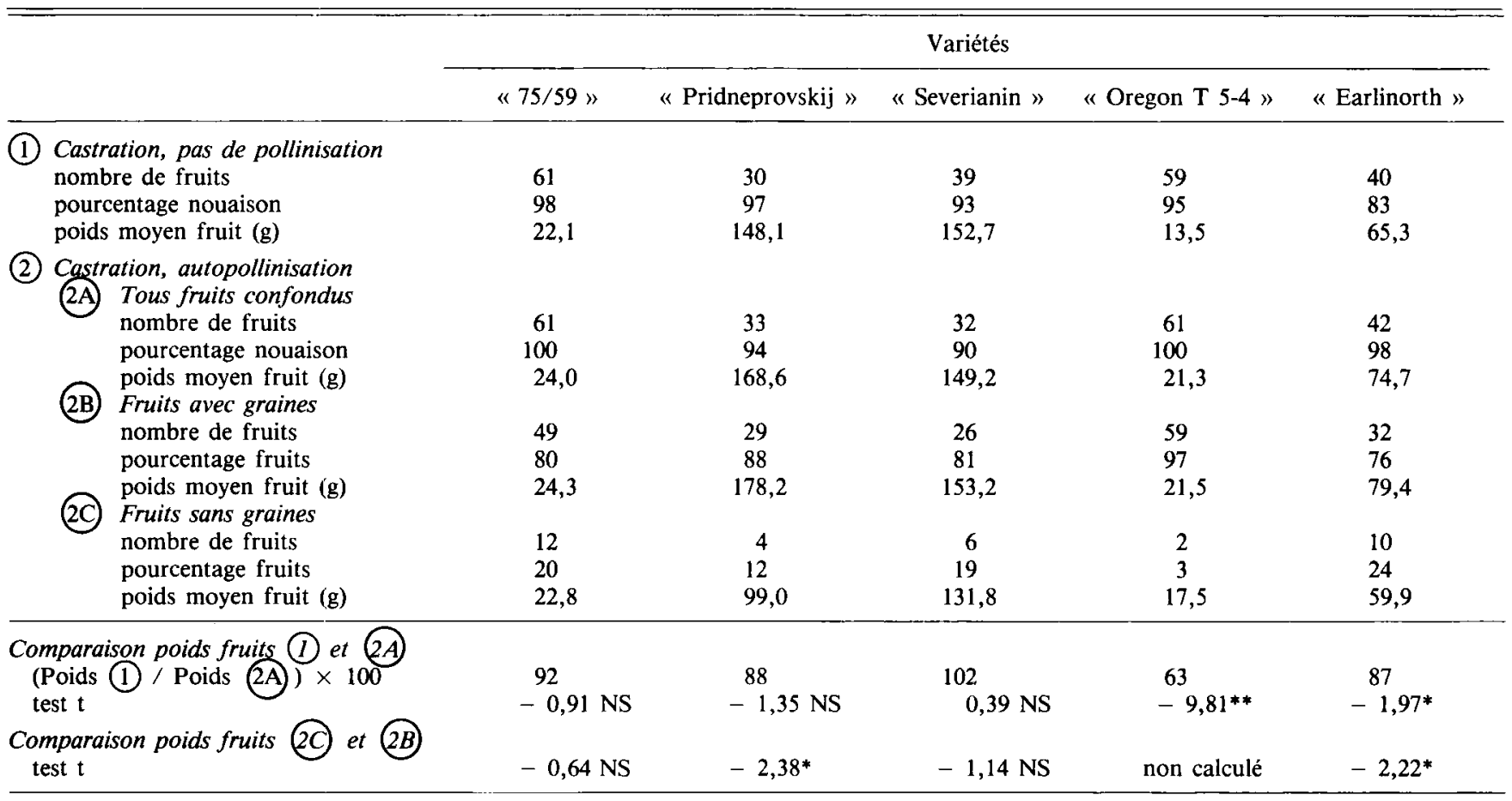

Tests $t$ de comparaison de moyennes.

NS : non significatif $;^{*}$ : significatif au seuil 0,$05 ; * *$ : significatif au seuil 0,01 .

TABLEAU 4

Essai 2. Culture de printemps, sous serre verre chauffée, en pleine terre. Période de castration : 3 au 28 mars.

Experiment 2. Spring cultivation, under a heated glasshouse, in soil. Period of emasculation : 3rd to 28th March.

\begin{tabular}{|c|c|c|c|c|c|}
\hline & \multicolumn{5}{|c|}{ Variétés } \\
\hline & «75/59» & "Lyconorma " & « Lycoprea » & « Severianin » & «Parteno» \\
\hline $\begin{array}{l}\text { (1) Castration, pas de pollinisation } \\
\text { nombre de fruits } \\
\text { pourcentage nouaison } \\
\text { poids moyen fruit }(\mathrm{g})\end{array}$ & $\begin{array}{l}87 \\
92 \\
21,5\end{array}$ & $\begin{array}{l}64 \\
97 \\
25,4\end{array}$ & $\begin{array}{c}70 \\
100 \\
31,7\end{array}$ & $\begin{array}{l}46 \\
71 \\
97,0\end{array}$ & $\begin{array}{l}33 \\
55 \\
47,9\end{array}$ \\
\hline $\begin{array}{l}\text { (2) Castration, autopollinisation } \\
\text { nombre de fruits } \\
\text { pourcentage nouaison } \\
\text { poids moyen fruit }(\mathrm{g}) \\
\text { nombre moyen graines/fruit } \\
\text { nombres extrêmes graines } / \text { fruit } \\
\mathrm{r}: \text { poids fruit/nombre graines }\end{array}$ & $\begin{array}{l}59 \\
98 \\
27,4 \\
34,4 \\
0-100 \\
0,93^{* *}\end{array}$ & $\begin{array}{l}62 \\
98 \\
57,6 \\
62,5 \\
1-170 \\
0,62^{* *}\end{array}$ & $\begin{array}{l}46 \\
96 \\
73,4 \\
52,4 \\
2-152 \\
0,80^{* *}\end{array}$ & $\begin{array}{c}50 \\
85 \\
111,8 \\
22,6 \\
0-118 \\
0,49^{* *}\end{array}$ & $\begin{array}{l}51 \\
78 \\
68,9 \\
19,6 \\
\quad 0-87 \\
0,71^{* *}\end{array}$ \\
\hline $\begin{array}{l}\text { Comparaison poids fruits (1) et (2) } \\
\text { (Poids (1) / Poids (2) } \times 100 \\
\text { test } \mathrm{t}\end{array}$ & $\begin{array}{l}78 \\
-3,70^{* *}\end{array}$ & $\begin{aligned} & 44 \\
- & 10,21^{* *}\end{aligned}$ & $\begin{array}{l}43 \\
-11,35^{* *}\end{array}$ & $\begin{array}{c}87 \\
-2,01 *\end{array}$ & $\begin{array}{c}70 \\
-3,35^{* *}\end{array}$ \\
\hline
\end{tabular}

Coefficients de corrélation $r$ et tests $t$ de comparaison de moyennes :

NS : non significatif $;^{*}$ : significatif au seuil 0,$05 ;{ }^{* *}$ : significatif au seuil 0,01 .

NB : Les résultats concernant la variété «Sub-Arctic Plenty », qui n’a donné que des fruits minuscules à la suite du traitement sans pollinisation, ne sont pas reportés dans ce tableau.

différent statistiquement de celui des fruits obtenus avec pollinisation ; il n'y a aucune corrélation entre le poids des fruits obtenus avec pollinisation et le nombre de graines qu'ils contiennent. Cependant, dans l'essai 2, l'aptitude à la parthénocarpie de "Severianin » est moins marquée : à la suite du traitement sans pollinisation, le pourcentage de fruits noués et le poids de ces fruits sont inférieurs à ceux des fruits obtenus après pollinisation ; en outre, chez ces derniers, il y a une corrélation positive significative au seuil 0,01 entre le poids des fruits et leur nombre de graines. 
TABLEAU 5

Essai 3. Culture de printemps, sous serre verre chauffée, en pots. Période de castration : 27 mars au 17 avril. Experiment 3. Spring cultivation, under a heated glasshouse, in pots. Period of emasculation:27th March to 17th April.

\begin{tabular}{|c|c|c|c|c|c|}
\hline & \multicolumn{5}{|c|}{ Variétés } \\
\hline & $\ll 75 / 59 »$ & «Severianin » & «Parteno » & «Montfavet $191 »$ & «Monalbo » \\
\hline \multicolumn{6}{|l|}{ (1) Castration, pas de pollinisation } \\
\hline pourcentage nouaison & 98 & 70 & 82 & 97 & 100 \\
\hline poids moyen fruit $(\mathrm{g})$ & 35,7 & 128,9 & 69,2 & 44,3 & 30,8 \\
\hline \multicolumn{6}{|l|}{ (2) Castration, pollinisation } \\
\hline nombre de fruits & 57 & 23 & 36 & 47 & 34 \\
\hline pourcentage nouaison & 98 & 73 & 84 & 98 & 100 \\
\hline poids moyen fruit $(\mathrm{g})$ & 43,2 & 128,7 & 107,2 & 51,6 & 105,3 \\
\hline nombre moyen graines/fruit & 37,9 & 40,0 & 40,6 & 4,5 & 79,7 \\
\hline nombres extrêmes graines/fruit & $4-116$ & $3-122$ & $1-124$ & $0-15$ & $10-151$ \\
\hline$r:$ poids fruit/nombre graines & $0,56^{* *}$ & $-0,05 \mathrm{NS}$ & $0,50^{* *}$ & $0,44^{* *}$ & $0,53^{* *}$ \\
\hline \multicolumn{6}{|l|}{ Comparaison poids fruits (1) et (2) } \\
\hline (Poids (1) / Poids (2)) $\times 100$ & 83 & 100 & 65 & 86 & 29 \\
\hline test $\mathrm{t}$ & $-5,19^{* *}$ & $0,01 \mathrm{NS}$ & $-4,76^{* *}$ & $-3,17^{* *}$ & $-29,63^{* *}$ \\
\hline
\end{tabular}

Coefficients de corrélation $r$ et tests $t$ de comparaison de moyennes :

NS : non significatif ; ${ }^{*}:$ significatif au seuil 0,$05 ; * *$ : significatif au seuil 0,01 .

- «Montfavet $191 »$ (essai 3 ; tabl. 5). Sans pollinisation, cette lignée donne des fruits dont le grossissement est précoce, et de même apparence (forme, couleur) mais plus petits que les fruits obtenus après pollinisation. Bien que ces derniers ne renferment que peu de graines $(4,5$ en moyenne), le coefficient de corrélation entre le poids de ces fruits et leur nombre de graines est significatif au seuil 0,01 .

- «75/59» (essais $1,2,3$; tabl. 3, 4, 5). Si cette lignée donne dans l'essai 1 des fruits de même grosseur à la suite des traitements avec et sans pollinisation, les fruits obtenus sans pollinisation sont significativement plus petits que ceux obtenus après pollinisation dans les essais 2 et 3 . Dans ces 2 derniers essais, la corrélation entre le poids des fruits issus de pollinisation et leur nombre de graines est significative au seuil 0,01 . Notons cependant que dans les 3 essais, on ne peut distinguer les fruits issus des 2 traitements ni par leur forme, ni par leur couleur, et que le grossissement de l'ovaire des fleurs non pollinisées est précoce.

- "Pridneprovskij» (essais 1 et 4 ; tabl. 3 et 6 ). Le grossissement des ovaires des fleurs castrées non pollinisées a été beaucoup plus tardif que celui des ovaires des fleurs pollinisées. A maturité, les fruits du traitement sans pollinisation ont le même aspect (forme, couleur) que ceux du traitement avec pollinisation; ils sont légèrement plus petits, cependant la différence n'est pas significative au seuil 0,05 . Notons que, à l'intérieur du traitement avec pollinisation, les fruits sans graines sont significativement (au seuil $0,05)$ plus petits que les fruits avec graines.

- "Earlinorth" (essai 1 ; tabl. 3). Sans pollinisation, "Earlinorth » développe des fruits moins nombreux et plus petits qu'après pollinisation.

- «Parteno » (essais 2 et 3 ; tabl. 4 et 5 ). Sans pollinisation, "Parteno » développe tardivement des fruits moins nombreux (essai 2), plus petits (essais 2 et 3) qu'après pollinisation ; leur aspect est différent.

— «Oregon T 5-4» (essai 1 ; tabl. 3). Les fruits obtenus sans pollinisation sont significativement plus petits que ceux obtenus après pollinisation.

- «Lyconorma " et "Lycoprea" (essai 2 ; tabl. 4). Le poids des fruits obtenus sans pollinisation n'est que de 43 à 44 p. 100 de celui des fruits obtenus après pollinisation. Ces fruits se développent tardivement.

- «Sub-Arctic Plenty » (essai 2 ; tabl. 4) n'a développé que très tardivement des fruits très petits qui n'ont pas été récoltés, à la suite du traitement sans pollinisation.

— «Monalbo" (essai 3 ; tabl. 5), variété témoin non parthénocarpique a, à la suite du traitement sans pollinisation, donné 100 p. 100 de fruits noués ; mais ces fruits sont très petits $(29$ p. 100 du poids des fruits avec graines obtenus après pollinisation par "Apédice »), ils se développent tardivement, ils sont côtelés, alors que les fruits obtenus après pollinisation sont parfaitement lisses.

\section{DISCUSSION ET CONCLUSION}

La méthode utilisée ici pour mesurer l'aptitude à la parthénocarpie peut être critiquée. En effet ce que nous mesurons est l'aptitude à développer des fruits parthénocarpiques à partir de fleurs débarrassées de leurs étamines avant la maturité du pollen. Or plusieurs auteurs ont souligné le rôle des jeunes étamines sur le développement de l'ovaire. Ainsi Hatcher (1945) a montré que, chez le riz, les anthères contiennent avant maturité des quantités considérables d'auxines, beaucoup plus que chez les anthères mûres. De même chez le pommier, le fait d'enlever de jeunes étamines, riches en pollen, a une incidence défavorable sur la croissance de l'ovaire avant l'anthèse (MURNEEK, 1954). A contrario, on peut aussi se demander si la castration proprement dite n'est pas capable d'induire un certain développement parthénocarpique 
TABLEAU 6

Essai 4. Culture de printemps, sous serre verre chauffée, en pots. Période de castration : 12 avril au 2 mai.

Experiment 4. Spring cultivation, under a heated glasshouse, in pots. Period of emasculation : 12th April to 2 nd May.

\begin{tabular}{|c|c|}
\hline & Variété \\
\hline & «Pridneprovskij » \\
\hline $\begin{array}{l}\text { (1) Castration, pas de pollinisation } \\
\text { nombre de fruits } \\
\text { pourcentage nouaison } \\
\text { poids moyen fruit (g) }\end{array}$ & $\begin{array}{l}66 \\
94 \\
99,6\end{array}$ \\
\hline 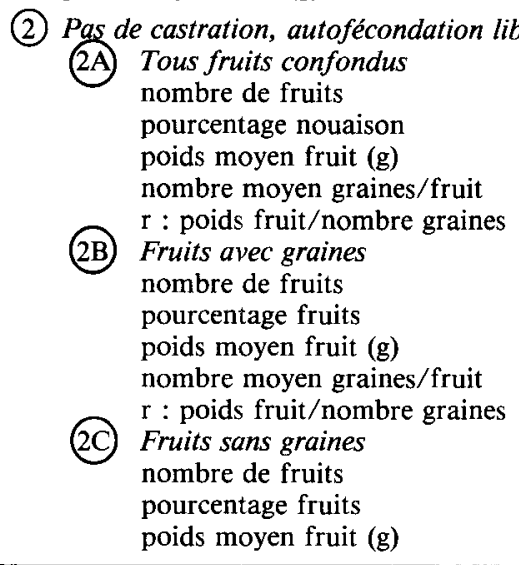 & $\begin{array}{c}34 \\
79 \\
117,1 \\
45,8 \\
0,51^{* *} \\
23 \\
68 \\
131,7 \\
67,7 \\
0,42^{*} \\
11 \\
32 \\
86,5\end{array}$ \\
\hline $\begin{array}{l}\text { Comparaison poids fruits (1) et } 2 A \\
\text { (Poids (1) / Poids (2A) } \times 100 \\
\text { test } \mathrm{t} \\
\begin{array}{l}\text { Comparaison poids fruits } 2 \mathrm{C} \text { et } 2 B \\
\text { test } \mathrm{t}\end{array}\end{array}$ & $\begin{array}{l}85 \\
-1,92 \mathrm{NS} \\
-2,39^{*}\end{array}$ \\
\hline
\end{tabular}

Coefficients de corrélation $r$ et tests $t$ de comparaison de moyennes :

NS : non significatif $;^{*}$ : significatif au seuil 0,$05 ;^{* *}$ : significatif au seuil 0,01 .

du fruit, par le biais de blessures du pistil. En fait, il est vraisemblable que les différentes variétés réagissent de façons différentes aux effets contradictoires de l'enlèvement de jeunes étamines sur le développement de l'ovaire : effet défavorable en supprimant une source d'auxines, effet favorable en provoquant une excitation par le biais d'une blessure du pistil.

Une comparaison portant sur l'aspect extérieur et la taille de fruits sans graines et de fruits avec graines, obtenus en fécondation libre, serait moins critiquable. Cette comparaison a été faite dans le cas de la variété « Pridneprovskij » dans l'essai 4 (tabl. 6), où un tiers des fruits obtenus en fécondation libre, dans des conditions de culture cependant favorables à la nouaison (culture sous serre verre, floraison $2^{\text {e }}$ quinzaine d'avril), ne contenaient aucune graine. Cependant ces fruits parthénocarpiques étaient significativement plus petits que les fruits avec graines. Les conclusions que l'on peut tirer de cet essai quant à l'aptitude à la parthénocarpie de "Pridneprovskij », ne sont pas différentes de celles que l'on peut tirer de l'essai 1 (tabl. 3).

La comparaison entre fruits avec ou sans graines obtenus en fécondation libre n'est possible qu'à condition qu'un pourcentage suffisant de fruits sans graines soit disponible. C'est rarement le cas en conditions de milieu favorables à la formation du pollen, à la pollinisation et à la fécondation. Il faudrait donc se placer dans des conditions moins favorables, de façon à ce que les variétés puissent exprimer leur aptitude à la parthénocarpie. C'est par exemple le cas en conditions de températures extrêmes (basses ou élevées).

Les différences dans les méthodes utilisées expliquent très certainement les différences dans les résultats. Ainsi "Oregon T 5-4 " n'a montré, si l'on en juge par la taille des fruits, qu'une aptitude limitée à la parthénocarpie dans nos essais, à la suite du traitement avec castration sans pollinisation, alors que selon BAGGETT \& FRAZIER (1978), cette variété réagit aux températures froides par la formation de fruits parthénocarpiques; cependant ces auteurs ne précisent pas la taille relative des fruits avec et sans graines. "Sub-Arctic Plenty », qui n'a montré aucune tendance à la parthénocarpie dans nos essais, donne des fruits parthénocarpiques à températures élevées (EL A HMADI \& STEVENS, 1979). Dans ces exemples, il est difficile de savoir si les températures extrêmes agissent directement sur le déclencheinent du développement parthénocarpique des fruits, ou si elles agissent indirectement, en empêchant la pollinisation et en révélant une aptitude latente à la parthénocarpie, aptitude qui ne serait pas révélée après un traitement à température normale faisant intervenir une castration non suivie de pollinisation.

Chez aucune des variétés testées, nous n'avons noté de différence dans l'aptitude à la parthénocarpie selon l'étage des inflorescences. Ce résultat est en désaccord avec ceux de LUDNIKOVA (1970) en ce qui concerne " Pridneprovskij»: en effet, d'après LUDNIKOVA, 
après castration des fleurs non suivie de pollinisation, des fruits parthénocarpiques peuvent se développer sur les premiers bouquets de "Pridneprovskij", jamais sur les $4^{\mathrm{e}}$ et $5^{\mathrm{e}}$ bouquets.

Le critère que nous avons utilisé (comparaison de fruits issus d'un traitement avec castration sans pollinisation et de fruits obtenus avec pollinisation) est particulièrement sévère. Les variétés qui montrent un niveau élevé de parthénocarpie dans ces conditions méritent d'être étudiées plus avant. Plusieurs autres critères sont apparus intéressants pour juger de l'aptitude à la parthénocarpie d'une variété :

- la précocité et la rapidité du grossissement parthénocarpique de l'ovaire,

- le pourcentage de fruits noués,

- la grosseur des fruits,

- pour les traitements avec pollinisation, l'existence ou non d'une corrélation entre le poids des fruits et le nombre de graines qu'ils contiennent,

- enfin l'aspect extérieur des fruits (côtes plus ou moins marquées, aspect brillant ou terne).

En tenant compte de ces critères, les variétés étudiées peuvent être regroupées de la manière suivante :

(1) Aptitude à un développement précoce de fruits sans graines de même présentation que les fruits avec graines et de taille comparable. D'après les résultats présentés ici, "Severianin » (essais 1 et 3 ) et «75/59 » (essai 1) peuvent être classés dans cette catégorie. Les pourcentages de nouaison à la suite des traitements avec et sans pollinisation sont très voisins, il n'y a pas de corrélation entre le poids des fruits et leur nombre de graines, enfin il est impossible de distinguer les fruits avec ou sans graines d'après leur aspect extérieur.

(2) Aptitude à un développement précoce de fruits sans graines de même présentation mais significativement plus petits que les fruits avec graines. C'est le cas de "Severianin " (essai 2), "Montfavet 191 " (essai 3) et «75/59» (essais 2 et 3 ), où l'on trouve une corrélation significative au seuil 0,01 entre le poids des fruits et le nombre de graines.

(3) Aptitude à un développement de fruits sans graines plus petits que les fruits avec graines et de présentation différente (plus côtelés, plus ternes). Le grossissement parthénocarpique de l'ovaire est tardif. Peuvent être classés dans cette catégorie "Pridneprovskij " (essais 3 et 4), "Earlinorth » (essai 3 ) et « Parteno » (essais 2 et 3) ; ainsi que "Oregon T 5-4 " (essai 1), «Lyconorma » (essai 2) et "Lycoprea » (essai 2) dont l'aptitude à la parthénocarpie est encore moins marquée que celle des 3 variétés précédentes.

(4) Aptitude nulle ou quasi nulle à développer des fruits parthénocarpiques. "Monalbo " (essai 3) ne développe que très tardivement de très petits fruits, très côtelés ; il en est de même pour "Sub-Arctic Plenty » (essai 2) dont les fruits n'ont pas même été récoltés.

Ainsi, seuls dans nos essais, "Severianin", «Montfavet 191 » et «75/59 " ont été capables, à la suite d'un traitement faisant intervenir une castration non suivie de pollinisation, de développer précocement des fruits parthénocarpiques de même présentation - quoique parfois plus petits - que les fruits avec graines obtenus après pollinisation.

Les variétés "Pridneprovskij », " Earlinorth », "Parteno ", et dans une moindre mesure "Oregon T 5-4 ", "Lyconorma » et « Lycoprea " se sont montrées capables, dans nos essais, d'exprimer une certaine aptitude à la parthénocarpie, mais les fruits sans graines qu'elles développent sont plus petits, plus tardifs et de présentation moins régulière que les fruits avec graines. Le niveau de parthénocarpie des variétés de ce groupe présente un intérêt limité du fait de l'existence de variétés dont l'aptitude à la parthénocarpie est plus marquée.

Les 3 variétés "Severianin ", " Montfavet 191 » et «75/59 » méritent une étude plus approfondie, en particulier une étude de la manifestation de l'aptitude à la parthénocarpie dans différents milieux et, par conséquent, de leur intérêt dans un programme de sélection. Il convient en effet de noter dès maintenant que, même dans des conditions favorables et somme toute très voisines (culture de printemps sous serre verre chauffée), les 2 variétés "Severianin " et «75/59 », qui ont été étudiées chacune dans 3 essais différents, ont montré une aptitude à la parthénocarpie variable d'un essai à l'autre (si l'on en juge par le pourcentage de nouaison et le poids des fruits sans graines). Les conditions de milieu doivent donc jouer un rôle important sur l'expression de l'aptitude à la parthénocarpie de ces variétés.

Reçu le 11 avril 1984 Accepté le 31 juillet 1984.

\section{RÉFÉRENCES BIBLIOGRAPHIQUES}

Baggett J. R., Frazier W. A., 1978. Oregon T 5-4 parthenocarpic tomato line. Hortscience, 13 (5), 599.

Dovedar S. A., 1973. Etudes des particularités de la mise à fruit des tomates soumises à des températures élevées. Mise au point de méthodes pour améliorer cette mise à fruit (en russe). Résumé de thèse présentée à l'Académie d'Agriculture Timiriazev de Moscou, $16 \mathrm{p}$.

El Ahmadi A. B., Stevens M. A., 1979. Genetics of high temperature fruit set in the tomato. J. Am. Soc. Hortic. Sci., 104 (5), 691696.
Harris R. E., 1972. Three new Sub-Arctic type tomatoes : Early Sub-Arctic, Sub-Arctic Midi and Sub-Arctic Plenty. Can. J. Plant Sci. $52,119-120$.

Hatcher E. S. J., 1945. Studies in the vernalisation of cereals. IX. Auxin production during development and ripening of the anther and carpel of spring and winter rye. Ann. Bot., 9 (35), 235-266.

Ludnikova L., 1970. Parthénocarpie chez la tomate (en russe). Kishinev, Moldavian SSR, Kartja Moldovenjaske, 98 p. 
Murneek A. E., 1954. The embryo and endosperm in relation to fruit development, with special reference to the apple, Malus sylvestris. Proc. Am. Soc. Hortic. Sci., 64, 573-582.

Paszkowska I., 1982. Occurence of parthenocarpy in self pruning tomato cultivars. 2Ist Int. Hortic. Congr., Hamburg, Fed. Repub. of Germany, August-September 1982, 1446 (Abstr.).

Pecaut P., Philouze J., 1978. A sha pat line obtained by natural mutation. Rep. Tomato Genet. Coop., 28, 12.

Philouze J., 1983. Parthénocarpie naturelle chez le tomate. I. Revue bibliographique. Agronomie, 3 (7), 611-620.
Preil W., 1973. Zur Parthenokarpie bei Tomaten in Abhängigkeit vom Temperaturverlauf. Angew. Bot., 47, 135-140.

Preil W., Reimann-Philipp R., 1969. Untersuchungen über die Einflüsse verschiedener Umweltfaktoren auf die Funktionsfähigkeit der Pollen von Tomaten. Angew. Bot., 43, 175-193.

Reimann-Philipp R., Fuchs G., 1969. Verfrühung der Tomatenernte unter Glas durch Verwendung von Zusatzlicht und speziell selektierten Genotypen Buschtomaten. Erwerbsgärtner, 23, 2153-2155.

Soressi G. P., Salamini F., 1975. A monomendelian gene inducing parthenocarpic fruits. Rep. Tomato Genet. Coop., 25, 22. 